\title{
A Location Service for Partial Spatial Replicas Implementing an R-Tree in a Relational Database
}

\author{
Yun Tian \\ Department of Computer Science, \\ Eastern Washington University \\ Cheney, WA, USA 99004 \\ Philip J. Rhodes \\ Department of Computer and Information Science, \\ University of Mississippi, \\ University, MS, USA 38677
}

\begin{abstract}
As parallel computing has become increasingly common, the need for scalable and efficient ways of storing and locating data has become increasingly acute. For years, both grid and cloud computing have distributed data across machines and even clusters at different geographic locations (sites). However not all sites need all of the data in a particular data set, or have the (perhaps specialized) processing capabilities required. These facts challenge the conventional wisdom that we should always move the computation to the data rather than the data to the computation. Sometimes the data actually required is small. In other cases, the site with specialized processing capabilities (such as a GPU equipped cluster) cannot handle the demands placed on it unless a way is found to let that cluster select the data that is actually needed, even if it is not stored locally.

Keywords: Globus Toolkit, Replica Location Service, R-tree, Prefetching, Spatial, Replica
\end{abstract}

Email addresses: ytian@ewu.edu (Yun Tian), rhodes@cs.olemiss.edu (Philip J. Rhodes)

Preprint submitted to Journal of Parallel and Distributed Computing December 29, 2015

(C) 2016. This manuscript version is made available under the Elsevier user license http://www.elsevier.com/open-access/userlicense/1.0/ 


\section{Introduction}

Although the problems just described are general, this paper concentrates on the problem of computation with spatial data. Such data is often the product of large computations and simulations, and also natural observations. In any case, spatial data sets present special problems, including not only size but also the mismatch between the $n$-dimensional nature of the dataset and its representation on disk. Because of the size of the datasets, it is also very likely that the original data will be copied and broken up into partial replicas that will be moved to the sites that require them.

An essential task, then, is to keep track of the many locations of the partial spatial replicas. The partial spatial replica location service (SRLS) does exactly that, from within the Globus toolkit, in each case using a relational data base (RDBMS).

Our replica location service identifies the set of partial spatial replicas that intersect with a region of interest, an important component of a large scale distributed storage system and for partial replica selection.

This paper describes and compares two different implementations of an SRLS, both using a relational database to store replica locations. We also offer suggestions for future work that would be applicable to a third implementation.

Replication and Replica Selection are widely used in distributed systems to help distribute both data and the computation performed upon it. It also increases reliability. The Globus Toolkit includes a Replica Location Service $(R L S)$ that provides users with a flexible mechanism to discover replicas that are convenient for a particular computation [1][2][3][4][5]. By providing multiple copies of portions of the same dataset, partial replicas can increase both I/O bandwidth and options for scheduling computation.

Recently, we have been working on the problem of partial replica selection for spatial data. A spatial dataset associates data values with locations in an $n$-dimensional domain and is commonly used in various fields of engineering and the sciences. For example, a climatologist might use spatial data produced by simulation to predict temperature changes for the Gulf of Mexico over a long time period. An agronomist might use spatial data to represent soil humidity for a large tract of land. The size of spatial datasets is growing rapidly due to advances in measuring instrument technology and more accessible but less expensive computational power.

Partial spatial replicas are incomplete portions of a larger spatial dataset. 
In grid computing, partial replicas result from research collaboration needs. For example, scientists may download subsets of a large data volume onto a local computer, thus creating a large number of partial replicas in a grid.

Several factors motivate us to choose among partial spatial replicas in grid. Partial spatial replicas on different grid node may have different storage organization, i.e. the order in which we linearize the high-dimensional data into one-dimensional physical storage. Furthermore, disk and network performance parameters on a grid node also influence the time cost of accessing replicas on that node. Thus it is crucial to decide from which grid node we should retrieve the replicas needed in computations.

The Geographical Information Systems (GIS) community has been actively investigating distributed data access for some time [6][7][8]. GIS data generally refers to the surface of the earth, and is usually two dimensional. Information such as road locations, municipal boundaries, bodies of water, etc. [9], can be represented using a sequence of points denoting the feature. Although this type of data is certainly spatial, this paper focuses on volumetric data. For example, Computational Fluid Dynamics (CFD) simulations commonly represent three or four dimensional volumes using rectilinear grids of data points that span the volume. Conceptually, such datasets (and subsets extracted from them) often have the shape of a rectangular prism.

The work described here was performed within the context of the Granite component of the The Distributed Spatial Computation and Visualization Environment (DISCoVer), which provides efficient access to spatial datasets stored on local or remote disks [10][11][12]. Granite allows its users to specify spatial subsets of larger $n$-dimensional volumes for retrieval. It takes advantage of UDT [13], a UDP based reliable data transfer protocol that is well suited to the transfer of large data volumes over high bandwidth-delay product connections. The combination of UDT and Granite provides fast access to subsets of datasets stored remotely. If a computation needs only a comparatively small subset of a larger volume, accessing that subset remotely may be much faster than moving the entire volume. The flexibility provided by this additional option is especially welcome in heterogeneous environments, where the hardware that best suits a computation (e.g. a GPU) might be located far from the dataset.

The Magnolia component of DISCoVer is intended to integrate Granite's unique spatial capabilities with existing Grid software. Replica selection for partial spatial replicas is an important part of Magnolia, and requires both a way to discover the partial replicas that intersect with a spatial query and 
a model of access time that allows Magnolia to choose between them. The Granite system already incorporates the concept of a storage model, which can be used to infer disk access costs. This paper concentrates on the problem of efficiently determining the set of partial replicas that intersect with the spatial query bounds given by the user.

It is important to note that although the implementations described here depend upon existing code in the DISCoVer and Globus tools, the problems we are solving are applicable for any system designed to handle distributed spatial datasets, including cloud systems intended for large scientific datasets and computation.

The rest of the paper is organized as follows. Followed by section 2 describing our motivation and contributions, section 3 presents related work. In section 4, we further describe the problem that we are addressing. Section 5 describes and analyzes our first design of SRLS, the GTR-tree implementation. The MAQR-tree, RLS modification, and proposed RTP are presented in section 6 . In section 7 , the advantages and usefulness of proposed techniques are validated through experiments. We conclude by summarizing this work and pointing out future research directions in section 8 .

\section{Motivation and Contributions}

In grid computing, it is quite common that middleware uses a relational backend database for data storage. The primary goal of this work is to add fast indexing for high dimensional data in existing middleware, the Replica Location Service (RLS). RLS primarily consists of three components - client tools, dedicated remote service APIs and backend data storage - a design widely accepted in the scientific data management community[14]. Because of the need to preserve this three-tier architecture, we implement the R-tree on top of the relational database already used by RLS.

Our Spatial Replica Location Service (SRLS) is able to process large spatial queries in a grid, each query may involves thousands of replica IO operations. In a large grid, hundreds or thousands of such queries could be submitted to the server in parallel. A spatial query that covers several thousand replicas may execute in more than a second on the server. In addition, querying the Replica Location Service is usually the first step for a distributed file system or a replica selection module. The SRLS is frequently queried for finding all relevant replicas. Thus, its performance dramatically influences the entire performance of a distributed system. 
We identify two major contributions of this paper. First, to the best of our knowledge, this is the first proposal to implement a tree structure inside a relational database for metadata management. Using this design, complexity and development cycles of such a location service could be dramatically reduced by using the atomicity features in DBMS, while still providing high performance comparable to PostgreSQL. The transaction overhead of our design is not very significant.

The proposed system is useful when users cannot replace the RDBMS or modify its internals. In addition, to allow portability to different storage infrastructures, placing specialized functionality, such as the R-tree indexing, in the middleware offers more options for switching storage platforms.

Second, we proposed R-tree prefetching. Through experiments, we provide deeper understanding about why the R-tree prefetching is influenced by backend query aggregation and why it affects the effectiveness of Spacefilling curves. We believe R-tree prefetching could be easily adapted to other systems that rely on tree traversal operations.

\section{Related Work}

Replica location service and replica selection in grid computing has been addressed by many researchers. Chervenak et al. described the Giggle framework, including RLS requirements, implementation, and performance results [2]. Cai et al. proposed a Peer-to-Peer Replica Location Service(P-RLS) with the properties of self-organization, fault-tolerance and improved scalability [4]. More recently, Chervenak et al. systematically described Globus RLS framework design, implementation, performance and scalability evaluation, and its production deployments [15]. Rahman et al. proposed two different replica selection techniques: the $k$-Nearest Neighbor rule based on a file transfer log and file transfer time prediction using a neural network [16]. The $P U-D G$ Optibox package is proposed by Li et al.[17] to facilitate parallel download from a ranked list of grid nodes with higher download performance. GRESS, a grid replica selection service based on the Open Grid Services Architecture, is presented by Zhao et al., which unifies many replica selection methods into a generic platform [18]. Vazhkudai et al. [1] present a design and implementation of a high-level replica selection service, which uses information about replica location and user preferences.

In the GIS context, Wu et al. described a framework for a spatial data catalog implemented on top of a grid and P2P system [6]. Wei and Di et al. 
implemented a Grid-enabled catalogue service for GIS data, by adapting the Open Geospatial Consortium (OGC) Catalogue Service for Web Specification and the ebXML Registry Information Model (ebRIM) [7, 8]. The OGC publishes a number of standards for GIS databases and applications, but these aren't well suited for other types of spatial data, especially those that entail three or more dimensions. For this reason, databases such as PostGIS [19] that implement the OGC GIS standard are not easily applied to other types of scientific data or metadata.

Narayanan et. al. described a middleware GridDB-Lite which provides basic scientific database support for data-driven applications in the grid [20]. To expedite selection of the data of interest, they implemented an R-tree via summary index files and detailed index files in their indexing service. In another paper [21], Narayanan presented a generic framework to support partial data replication and data reordering. Weng et. al. described a partial replica selection algorithm for serving range queries on multidimensional datasets [22]. Our work differs from these efforts in two important respects. First, our MAQR-tree integrates an R-tree into the Globus toolkit to support spatial metadata operations. Second, we investigated the representation of an R-tree constructed in a relational database.

To manage spatial metadata for fast retrieval, we chose the R-Tree [23] data structure for our system from among several other spatial data structures, including the Quadtree, Octree, Kd-tree and UB-tree. We require a data structure which returns the collection of replica Minimal Bounding Rectangles(MBRs) that intersect with a query region. OLAP methods like the UB-tree [24] return a collection of points (records) that are contained within a query region, making them inconvenient for our purposes. As described by Kamel et al., Quadtrees and Octrees typically divide the spatial replicas into smaller blocks, thus generating more partial spatial replicas during construction of the tree [25]. Kd-trees [26] have a similar problem because they require us to divide the space with a splitting hyperplane. However, the R-tree and related methods (e.g. the X-tree [27]) allow for efficient retrieval of the replica MBRs that intersect with a query region, without splitting replicas into smaller pieces.

Section 6.3.1 describes our use of the Morton Space-filling curve, also known as z-ordering. The Morton Space-filling curve and Hilbert curve are widely used in multidimensional access methods [25, 28]. Faloutsos et al. compared the Hilbert curve and the Morton curve. Their work concluded that the Hilbert curve has better distance-preserving mapping than the Mor- 
ton curve, but the Hilbert curve is more complex to calculate [29]. Both the Morton and Hilbert curves are found in commercial database systems. Microsoft $S Q L$ server 2008 used the Hilbert curve to determine the value of cell identifier, thus maintaining good spatial locality [30]. Oracle has used the Morton curve in their products for some time [28]. TransBase, a relational database system, integrated the $U B$-Tree into its kernel to support spatial indexing. A UB-tree combines a B-Tree with the Morton curve, mapping multi-dimensional data to one-dimensional space using the Morton value of the data points [24]. In P2P systems, Ganesan et al. [31] used the Morton curve to reduce multi-dimensional data to one dimension, then partitioned the dataset according to contiguous Morton value range. We conclude that these systems indicate no biased favor for Hilbert curve, even though it is slightly better distance-preserving. Because the Morton value can be simply computed by bit-interleaving, in this paper we use the Morton curve to re-order the 3D replicas and then construct the MAQR-tree.

The MAQR-tree representation in a relational database uses the concept of data-linearization [32][33]. In order to prefetch pointer-linked data structures, Luk et al. proposed two solutions: data-linearization prefetching and jump pointers. Data-linearizaion prefetching removes pointers by arranging data in a contiguous segment of memory. The Cache Sensitive $B+-$ Tree $(C S B+$ Tree $)$ employed the same idea, having only one pointer to a group of contiguous child nodes in memory [34]. In this paper, we use integer keys as references to a tree node and store the sibling nodes contiguously in a database table, but allow tree updates as described in section 6.3.2. This design is very suitable for prefetching.

I/O Prefetching is an important technique for improving performance by overlapping data access and computation costs. Its effectiveness is greatly enhanced when guided by advance knowledge of what data is needed, then loading the data before they are accessed. Patterson et al. describe Informed Prefetching, in which application-disclosed access patterns(hints) are used to prefetch blocks [35]. Rhodes et al. describe Iteration Aware Prefetching in which the application uses iterators to describe the access pattern to underlying middleware, which then performs prefetching on behalf of the application [10][11].

Tree prefetching has been investigated in many works, but mainly focuses on the trees that reside in main memory [36][37][38][39]. Chen et al. evaluated prefetching $B^{+}$-Trees $\left(p B^{+}\right.$-Trees), which uses prefetching to effectively create wider tree node that could occupy multiple cache lines. In 
$\mathrm{pB}^{+}$-Trees, these cache lines comprising a wide tree node are prefetched in parallel with the first cache line in the tree node, thus hiding the latency of cache misses [36]. Kang et al. described R-Tree prefetching for the SPR-tree (Selective Prefetching $R$-tree) [38], which used an idea similar to the $\mathrm{pB}^{+}$Trees. Namely, the SPR-tree prefetches all the cache lines that comprise the wider tree node for an R-tree in main memory. In order to optimize both cache and I/O performance, in another paper Chen et al. proposed the fractal prefetching $B^{+}$-Trees ( $f p B^{+}$-Trees). The $\mathrm{fpB}^{+}$-Trees embed cache-optimized trees( $\mathrm{pB}^{+}$-Trees $)$within disk-optimized trees [37]. Kim et al. described fast architecture sensitive binary tree search on modern CPUs and GPUs, in which they use prefetching to overlap the memory access and computation for different queries [39].

Existing work primarily focuses on speculative or (uninformed) tree prefetching using one CPU sequentially. For example, when an internal tree node is visited, all its siblings are prefetched into cache, which may or may not be useful for subsequent queries. In contrast, our work adopts an informed tree-prefetching strategy using two threads in parallel. During tree breadthfirst search, we use a queue structure to store references to tree nodes that we will have to check against subsequently. A prefetching thread takes advantage of the references in the queue, and prefetches the entire tree nodes in advance, which guarantees to be visited for the current query. Meanwhile, a processing thread performs intersection test on the tree nodes that have been prefetched from hard disk.

Out-of-core tree traversal has been explored by many researchers. Lindstrom used out-of-core techniques with an octree for large surface meshes to visualize multi-resolution surfaces [40]. However, prefetching was not used during tree traversal. Sulatycke et al. used a tree traversal thread with several computation/visualization threads in order to hide disk latency costs, but also did not use prefetching during tree traversal [41]. The work described in this paper applies prefetching to the problem of out-of-core R-tree traversal when processing a spatial query.

\section{Algorithm Overview}

Before a computation begins in a grid, partial spatial replica selection must address two subproblems. The first subproblem is to efficiently identify the set of partial replicas that intersect the subvolume required by the computation in a distributed system. Second, from that set, we want to select 


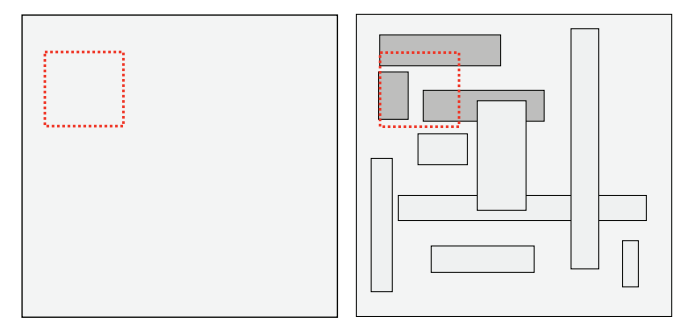

Figure 1: Intersected Replicas in 2D. The dotted square represents the spatial query, and intersected replicas are shown as shaded rectangles.

the "best" replicas to minimize transfer time. This requires a metric based on factors such as disk and network bandwidth and latency, data storage organization, etc. This paper focuses only on the first subproblem.

Figure 1 illustrates how the intersecting replicas are identified in the system. Spatial replicas and queries are commonly represented as MBRs, which approximate the extent of the object in space using minimum and maximum values for each dimension. In the $2 \mathrm{D}$ case, a spatial replica or query can be represented as $\left\{x_{\min }, y_{\min }, x_{\max }, y_{\max }\right\}$. These MBRs identify the region of the larger data space that each replica represents. Intersection tests are performed to determine which replicas intersect with the spatial query. In the worst case, each spatial replica in the catalog will be examined against the spatial query, which is very computationally expensive.

However, an $R$-tree can be used on each grid node to organize the metadata of all spatial replicas and to prune the search space. We define the out degree of a tree node as the actual number of child nodes. Each node of an R-tree can have a variable number of child nodes, up to a maximum value that we will refer to as the fanout. For our purposes, leaf nodes contain a reference to partial replicas, along with an MBR for each replica. Internal nodes contain references to child tree nodes, along with an MBR that encloses the MBRs of all the child nodes. During search, an internal node's MBR can be tested for intersection with the query rectangle, allowing large sections of the tree to be pruned.

$R L S$ in Globus Toolkit uses user-defined attributes, Logical File Names (LFNs), and Physical File Names (PFNs), to maintain the metadata of replicas. This metadata is stored in the $R L S$ backend's relational database. There are sets of tables in this database, including an $L F N$ table, PFN table, general attribute table, and attribute value tables for four types of attributes: 

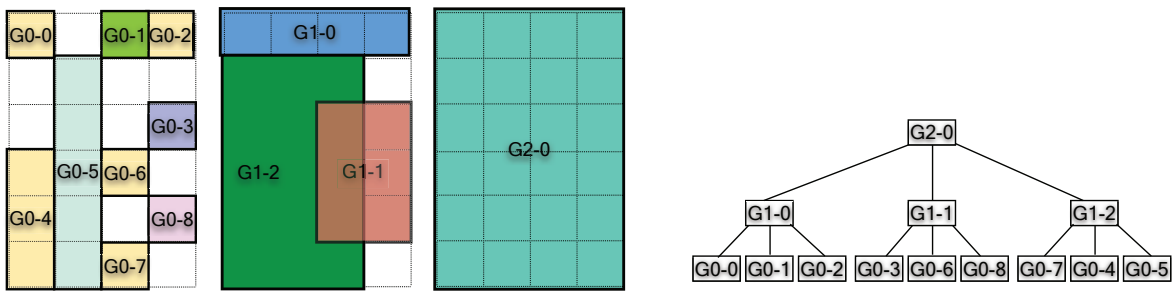

(a) Layout of 2D spatial replicas, grouped (b) Packed R-tree with out deinto tree nodes from left to right with left- gree $=3$ for figure (a). most the leaf nodes.

\begin{tabular}{|c|c|c|}
\hline ID & Attribute & Value \\
\hline “G0-0” & "MBR" & “0,0, I, I” \\
\hline "G0-0" & "Info" & $\begin{array}{l}\text { "<gtrtree> } \\
<\text { pfn }>\text { G0 } 0</ \text { pfn }><\text { numChild }>0<\text { /numChild }> \\
<\text { address>gsiftp://nodel.olemiss.edu/data/d0.bin</address }> \\
</ \text { gtrtree }>\text { " }\end{array}$ \\
\hline$\ldots \ldots$ & $\ldots \ldots$ & $\ldots \ldots$ \\
\hline “G2-0" & "MBR" & “0,0,4,6” \\
\hline “G2-0" & "Info" & 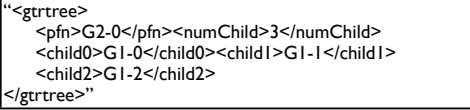 \\
\hline
\end{tabular}

(c) The GTR-Tree node representations for figure (b).

Figure 2: A GTR-tree on a single grid node.

string, int, float and date [3]. Users can submit a query on the client by specifying a $L F N$ or attribute value for the replicas, and the server will retrieve the metadata of all replicas that satisfy the query. In addition, users can create or add metadata for a replica or delete them from the catalogue.

\section{The First Design: The GTR-Tree}

Our first design is partially motivated by limitations in the existing RLS for partial spatial replicas.

\subsection{Limitations of $R L S$ for Spatial Replicas}

In our work [42], we identified two limitations when the Globus RLS was used for spatial data. First, the RLS server does not support spatial intersection queries efficiently because such queries are not implemented in the underlying relational database. Second, if we implement support for intersection queries using only the relational database, there is no mechanism for pruning the search, so each replica MBR must be examined for intersection 
with the query region. We surmised that implementing a spatial data structure on top of the existing RLS would greatly improve query performance by eliminating large sets of replicas from consideration.

To mitigate these RLS limitations, we implemented two important components on top of the existing Globus codebase. First, an $R$-tree was used to pack the metadata in the RLS backend relational database, allowing large numbers of replicas to be pruned from the search during an intersection query. We call our implementation a GTR-tree (Globus Toolkit R-Tree). Second, we used the RLS API to implement the intersection query routine, which is executed on the same machine as the RLS server. The query routine traverses the GTR-tree from root to leaf, finally returning the replicas that intersect with the query region. To enable distributed queries on the unmodified Globus Toolkit, users can submit arguments to the query routine by wrapping them in a Job Description Document(JDD) string used by GRAM, which will then invoke the query routine. The query results are transferred back to clients by using either a Java socket or GridFTP.

\subsection{The GTR-tree Representation}

The implementation of the GTR-tree [42] is shown in figure 2. To represent a GTR-tree in the Globus Toolkit RLS database, each tree node is described as a set $\{I D, M B R$, Info $\}$, where $I D$ is a PFN string repurposed to be a reference to the node, $M B R$ is a minimum bounding (hyper)rectangle that encloses all child MBRs, and In fo contains references to the child nodes. By associating $M B R$ and In $f o$ (two user-defined string attributes) with the PFNs, a GTR-tree node can be described using two rows in the table of RLS database.

In figure 2(c), the "ID" and "Attribute" columns are actually foreign keys referencing an integer PFN id and attribute id defined in two other tables. For simplicity and easy comprehension, we show the PFN name and the attributes name in that column.

\subsection{Limitations of The First Design}

The approach described above is still subject to some disadvantages. First, fanout of the GTR-tree is limited by the size of the string attribute in the RLS backend database. Although R-trees of large fanout can be represented in the database by introducing more user-defined attributes, we propose a more elegant solution in section 6.3.2. 
Second, GTR-tree node representation was not optimized and was constrained by the design of the existing table structure in the backend database. In particular, one tree node was stored in three different tables, which made the query and updates inefficient due to an expensive join operation.

Third, although the GTR-tree dramatically prunes the search space, some amount of metadata was still sent from RLS client code to the query routine via local socket, which incurs some overhead. Also, because we needed to use existing Globus tools, we incurred additional latencies associated with GRAM and GridFTP when performing a distributed query.

In GTR-tree implementation, the query routine works as a client for accepting spatial queries, implemented on top and outside of client APIs of existing Replica Location Service in Globus Toolkit (RLS). When a GTRtree query is submitted, R-tree nodes are transferred through many units, from the query routine, to RLS client, to RLS server, then to RLS backend database, during tree traversal. Although the query routine is located on the same grid node as a RLS does, the frequent data exchanges between the query routine and the RLS client use network sockets. On the other hand, in MAQR-tree, spatial query routines are integrated into modified RLS, thus become a subset of its client APIs.

\section{The Second Design: The MAQR-tree}

In this design, we modified the Globus Toolkit $R L S$ to support spatial metadata management in a grid [43]. We evaluated the performance of an $\mathrm{R}$-tree stored in a relational database, and some related factors which influence the performance of spatial queries or updates, including Fanout, Morton Space-filling Curve and Tree Node Representation. Our proposed RTP technique further improves the query performance.

\subsection{RLS in Globus Toolkit}

Communication between client and server in the Globus RLS uses a simple string-based $R P C$ protocol [4][44], which relies on the Grid Security Infrastructure(GSI) and the globus_io socket layer from the Globus Toolkit [2]. Method names, parameters and results are all encoded as null terminated strings.

An $R P C$ method invocation in the Globus RLS includes several steps. First, the client sends the method name and all the arguments to the server. A thread on the server searches through a method array for an element 

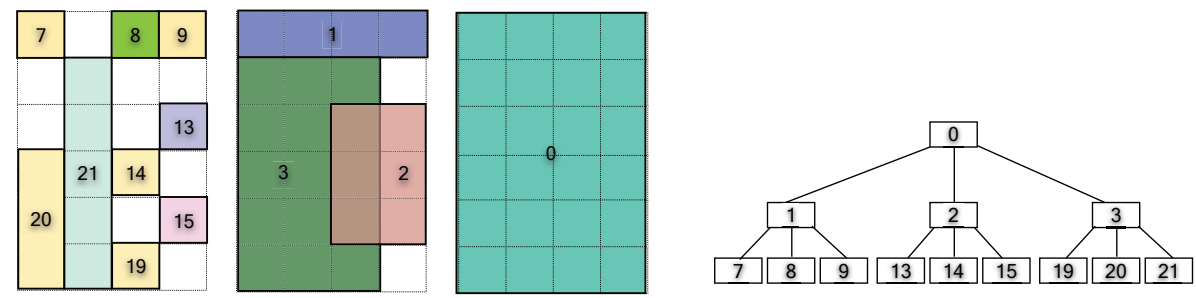

(a) Layout of 2D spatial replicas same as fig- (b) R-tree with fanout $=6$ and out ure $2(\mathrm{a})$. degree $=3$ for figure (a).

\begin{tabular}{|c|c|}
\hline $\begin{array}{c}\text { Node id } \\
\text { (int) }\end{array}$ & $\begin{array}{c}\text { Info } \\
\text { (varchar) }\end{array}$ \\
\hline 0 & "“<maqr $><$ numChild $>3</$ numChild $><$ MBR $>0046</$ MBR $><$ /maqr $>$ \\
\hline 1 & “" $<$ maqr $><$ numChild $>3<$ /numChild $><$ MBR $>0041</$ MBR $><$ /maqr $>$ \\
\hline & \\
\hline 21 & 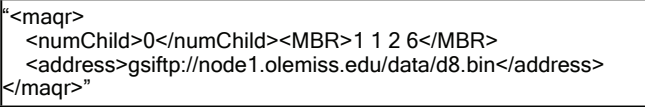 \\
\hline
\end{tabular}

(c) MAQR-Tree node representations for figure (b).

Figure 3: An MAQR-tree example on a single grid node. The number in each tree node indicates the node id.

matching the requested method name. After the matching method is invoked, execution results are sent back to the client. It follows that we can easily extend the functionality of the RLS by adding new entries to the method array, where each entry includes a method name and a reference to the code itself.

\subsection{Modifying RLS}

The second design extends the $R L S$ in Globus Toolkit 5.0.3 for spatial replica selection in several ways. First, we added a new table to the backend database, allowing all tree nodes to be stored in a single database table. Second, we added spatial replica query and insertion methods to the $R P C$ methods array on the server, to service the spatial requests from the clients. Third, we implemented new methods on the server to communicate with its backend database, where query aggregation is used. Fourth, we added the spatial replica query and insertion functionality to the RLS client tool, client $C A P I$ and Java API of the existing RLS. 


\subsection{The $M A Q R$-tree}

To distinguish from the GTR-tree implementation of an SRLS, we call the second design the MAQR-tree. Two critical factors influence tree performance. First, we consider how to select sets of MBRs to be siblings of a single parent, sometimes called clustering [25]. Ideally, sibling nodes would be nearby in space, and also stored close together in the underlying database to improve I/O performance. The space-filling curve is a useful tool for ordering child MBRs in a manner that addresses these concerns. The other critical factor is how to efficiently represent the tree structure in the RLS database. Reducing the storage volume for tree representation and improving access speed are both important goals here.

\subsubsection{Morton R-tree}

To construct the Morton R-tree, all spatial replicas are first sorted according to the Morton values of their center. Then we construct the $R$-tree in a bottom-up manner based on the specified fanout and the out degree of the tree node [45]. Replicas whose MBRs are associated with adjacent Morton values will be clustered into the same tree node. We evaluate the MAQR-tree performance with and without the Morton re-ordering in section 7 .

\subsubsection{MAQR-Tree Node Representation}

We added a new table named t_spatial_rep to the RLS backend database, to store the metadata of all spatial replicas. Two different table schemas have been designed, and the performances of two schemas are compared in section 7. Figure 3 describes one representation of the MAQR-tree. In order to emphasize different tree representations, replicas in figure 3 are grouped into tree nodes in a same way as in figure 2 .

Given a current node id pid, the tree fanout $f$, and out degree $d$, we can compute all its child ids using:

$$
\begin{array}{r}
f i r s t \_c h i l d \_i d=p i d \times f+1 \\
\text { last_child_id }=\text { first_child_id }+f-1 \\
\text { last_occupied_child_id }=\text { first_child_id }+d-1
\end{array}
$$

Also, given the child id cid, we can calculate its parent id pid by:

$$
p i d=\left\lfloor\frac{\text { cid }-1}{f}\right\rfloor
$$


To construct a R-tree with $n$ spatial replicas, node ids of the MAQR-tree on each level can be determined by using equation 1 and the tree height $H$, defined as length of the path from the root to the deepest node in the tree.

$$
H=\left\lceil\log _{d}(n)\right\rceil
$$

During tree construction, note that only $d$ replicas are clustered into the same tree node, the rest of $(f-d)$ unused node ids and all their descendant ids will be reserved for tree updates, with $d<=f$.

\subsubsection{Advantages of The MAQR-tree Representation}

There are several advantages associated with the MAQR-tree representation, relative to the GTR-tree and other designs.

- Node ID Representation: We used an integer to represent node id. In the underlying database, the performance is improved when tree nodes are retrieved using integer keys, especially after a relational database Index has been constructed on the column Node_id.

- Table Simplification: Metadata of spatial replicas is stored in a single table, and no relational join is performed during a query.

- No explicit representation of child and parent ids.: The storage size of the tree node is dramatically decreased, resulting in a more compact backend database, because no stored reference to the children is required. Instead we can calculate all child ids on the fly using equation 1.

- Arbitrarily large fanout values: The fanout of the MAQR-tree is no longer limited by the size of a table column. We can create a MAQRtree with a fanout of more than one thousand, for instance. Although a large number of node ids are reserved in the tree, the storage utilization is not influenced by the tree fanout.

- Query Aggregation: Database queries can be aggregated into a single database transaction using a range of tree node ids.

- Support for insertion and deletion: the MAQR-tree is not a Static Rtree. Instead it is dynamic, and allows insertion and deletion of replicas in the tree. 
- Highly recoverable and reliable: the MAQR-tree is stored on hard disk through underlying DBMS, which is not volatile after power is off. Also, we can easily export the entire tree by using built-in DBMS backup tools.

- Prefetching: We have developed a prefetching scheme that hides I/O costs as the tree is traversed.

\subsection{R-tree Prefetching}

The MAQR-tree is stored in the RLS's backend relational database. First, we used the existing storage infrastructure of the RLS. In addition, the existing RDBMS provides important functionality that makes it easy to implement. For example, the GTR-tree updates can use the atomic transaction mechanism of the underlying DBMS to maintain consistency when the server is simultaneously processing both queries and updates.

As described in section 3, RTP is based on the concept of applicationdisclosed access patterns. We traverse the R-tree using breadth-first search, which typically works with a queue structure. During traversal, processing one internal tree node may result in many enqueues of its children. This is especially true when query MBRs and the out degree of the R-tree are larger. These children in the queue can be considered as the prior knowledge of access patterns disclosed by the traversal, which we will certainly access in the future.

\subsubsection{Backend Query Aggregation}

To reduce the latency cost associated with each replica query, in prefetch thread we aggregate a group of replica reads into one larger read. We call

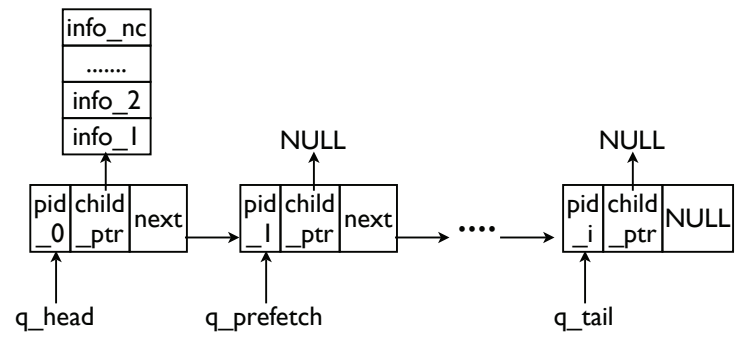

Figure 4: The queue structure used for R-tree prefetching with query aggregation. 
this technique backend query aggregation. Under the MAQR-tree representation, the prefetch thread first reads the tree node id pointed to by a prefetch pointer, and next computes the first_child_id and the last_child_id using equation 1. Then the prefetch thread will perform a range query on the table column Node_id shown in figure 3(c), and all child information is retrieved in one transaction. We constructed a relational index on the table column Node_id, so the range query on that column is fast. The next section presents RTP implemented with backend query aggregation.

\subsubsection{RTP Implementation}

The queue used in RTP is shown in figure 4. Each queue element has three fields. The field pid stores the tree node id whose MBR intersects with the query bound. The child_ptr field points to a chunk of memory storing all child information of the pid. The last field points to the next queue element. In addition, the entire queue structure has three pointers, q_head, q_prefetch, and q-tail.

Two threads are used for RTP, a traversal thread and a prefetch thread. When the queue contains at least one prefetched element, the traversal thread will dequeue an element and perform an intersection test on all children stored in the field child_ptr. It then enqueues the child ids whose MBRs intersect with the query MBR. When no prefetched elements are available, the traversal thread sleeps.

The children placed in the queue by the traversal thread will not yet have their information loaded from the database. When such elements exist, the prefetch thread will process the element indicated by the q-prefetch pointer, reading the child information into memory from the backend database for this node id. The prefetch thread then sets child_ptr to point to this memory and updates q-prefetch. When no queue element needs to be prefetched the prefetch thread sleeps.

Table 1: Experimental Grid Node Characteristics

\begin{tabular}{|c|c|c|c|c|c|c|c|c|}
\hline OS & Processor & Cores & Memory & $\begin{array}{c}\text { Java } \\
\text { version }\end{array}$ & $\begin{array}{l}\text { Globus } \\
\text { Toolkit }\end{array}$ & $\begin{array}{c}\text { unix- } \\
\text { ODBC }\end{array}$ & $\begin{array}{c}\text { MySQL } \\
\text { database }\end{array}$ & $\begin{array}{c}\text { MyODBC } \\
\text { library }\end{array}$ \\
\hline $\begin{array}{l}\text { Linux } \\
2.6 .18\end{array}$ & $\begin{array}{l}\text { Intel Xeon } \\
2.40 G \mathrm{GHz}\end{array}$ & 16 & $24 \mathrm{G}$ & $\begin{array}{c}\mathrm{SE} \\
1.6 .0 \_23\end{array}$ & $\mathrm{v} 5.0 .3$ & $\mathrm{v} 2.2 .11$ & $\mathrm{v} 5.0 .77$ & $\mathrm{v} 3.51 .26$ \\
\hline
\end{tabular}


Table 2: Spatial Extent of Representative Queries

\begin{tabular}{|c|c|c|c|}
\hline & 3D Query MBR (String) & Q5 & “200 200200400400 400” \\
\hline Q1 & "14001400 $1400140014001400 "$ & Q6 & “800 900100010001100 1400" \\
\hline Q2 & “1500 $15001600151015201640 ”$ & Q7 & “1024 1024102413241324 1324" \\
\hline Q3 & “505050100100 100” & Q8 & “160016001600 204820482048 ' \\
\hline Q4 & “100100100200200200” & $Q 9$ & "900 $9001000140011001400 "$ \\
\hline
\end{tabular}

Table 3: Number of Replicas Intersected with query in Grid

\begin{tabular}{|l|c|c|c|c|c|c|c|c|c|}
\hline & Q1 & Q2 & Q3 & Q4 & Q5 & Q6 & Q7 & Q8 & Q9 \\
\hline Average & 1995 & 2578 & 1288 & 3405 & 10667 & 15990 & 20181 & 22351 & 26523 \\
\hline Maximum & 2063 & 2667 & 1350 & 3534 & 10865 & 16221 & 20484 & 22493 & 26822 \\
\hline Minimum & 1911 & 2518 & 1243 & 3307 & 10527 & 15786 & 19976 & 22193 & 26347 \\
\hline
\end{tabular}

\section{Evaluation}

The Distributed Research Testbed (DiRT) is a multi-site instrument for developing and evaluating the performance of distributed software. We constructed a twenty three-node grid on DiRT using a version of Globus Toolkit 5.0.3 modified to include our spatial replica location service. In the grid, eight nodes are located at the University of Mississippi, seven of them at the University of Florida, five nodes at the University of Chicago and three nodes at the University of Hawaii. The characteristics of each grid node are described in table 1. We use unixODBC manager and MyODBC driver to communicate with MySQL server.

We used independent datasets on each grid node. One million 3D replicas were randomly generated on each grid node. One object randomly generates the center point of the 3D rectangle in the entire dataset domain (a $2048^{3}$ cube). Another Random object randomly generates the length of replicas along each axis, which is bounded in the range of 1 to $P$. The following tests use $P=512$, unless otherwise noted.

The total size of the metadata for one million replicas is $120 \mathrm{M}$. The average size of metadata for one replica takes 115 bytes, and the physical address string for each replica takes an average of 54 bytes. In the test, the physical address and the id of replicas are transferred back to the clients.

We carefully chose nine representative spatial queries in order to verify our system. Queries include one point query and eight rectangular queries of various sizes. The extent of these nine spatial queries, relative to the entire dataset (a $2048^{3}$ cube) are shown in table 2. For each query, table 3 shows 
Table 4: Average performance with different fanout and out degree choices of MAQR-tree

\begin{tabular}{|c|c|c|c|c|}
\hline Choices: & $\begin{array}{c}\text { Fanout=30 } \\
\text { Out degree=20 }\end{array}$ & $\begin{array}{c}\text { Fanout=70 } \\
\text { Out degree=50 }\end{array}$ & $\begin{array}{c}\text { Fanout=120 } \\
\text { Out degree=100 }\end{array}$ & $\begin{array}{c}\text { Fanout=220 } \\
\text { Out degree=200 }\end{array}$ \\
\hline R-tree without Morton Curve & $1.07 \mathrm{sec}$. & $0.95 \mathrm{sec}$. & $0.96 \mathrm{sec}$. & $1.55 \mathrm{sec}$. \\
\hline R-tree with Morton Curve & $0.84 \mathrm{sec}$. & $0.81 \mathrm{sec}$. & $0.81 \mathrm{sec}$. & $0.82 \mathrm{sec}$. \\
\hline
\end{tabular}

the average number (per grid node) of replicas that intersect that query, and the maximal and minimal number of replicas returned in the grid. All the following results were collected as an average of five runs to reduce the effect of filesystem caching, network behavior and other variables.

We performed experiments under two file caching conditions: a warm cache and a cold cache. A warm cache is defined as the state in which the file system has already cached the metadata that we are retrieving. The same query repeated more than once may be satisfied from the cache rather than from disk. For the cold cache case, we unmounted and remounted the file system where the RLS's backend database is stored, which discards file system cache contents. Also, we flushed all caches inside the MySQL server and restarted the SRLS server for each query.

We tested average performance under various MAQR-tree fanout and out degree values, as shown in table 4 . We observed that a MAQR-tree with out degree of 50 yields best performance on average. In the following tests, we used the MAQR-tree with out degree of 50, and fanout of 70 .

\begin{tabular}{|c|c|c|c|c|c|c|}
\hline Column Name: & id & x_min & y_min & x_max & y_max & address \\
\hline Type: & int & int & int & int & int & varchar \\
\hline
\end{tabular}

Figure 5: The schema used for the pure relational implementation, components of the MBR are stored as integers.

Table 5: Advantage of MAQR-tree query over pure relational

\begin{tabular}{|c|c|c|c|c|}
\hline & Data1 & Data2 & Data3 & Data4 \\
\hline Total Number & 1000000 & 1000000 & 1000000 & 1000000 \\
\hline Average number returned & 11645 & 4649 & 1816 & 2642 \\
\hline Average speedup & 1.9 & 4.36 & 7.75 & 8.13 \\
\hline
\end{tabular}


select address from $t$ spatial rep

where (not $\left(\left(x \_\min >q x_{\min }\right.\right.$ and $\left.x \_\min >q x_{\max }\right)$ or $\left.x \_m a x<q x_{\min }\right)$

and not $\left(\left(y \_\min >q y_{\min }\right.\right.$ and $\left.y \_\min >q y_{\max }\right)$ or $\left.\left.y \_\max <q y_{\min }\right)\right)$;

Figure 6: SQL statement for conducting spatial queries without a MAQR-tree. Used with the schema shown in figure 5, this SQL statement can retrieve replicas that intersect with a bounding rectangle $\left\{q x_{\min }, q y_{\min }, q x_{\max }, q y_{\max }\right\}$. Performance was found to be worse than the MAQR-Tree on average.

\subsection{Experimental Verification}

We performed a series of experiments to verify the usefulness of the MAQR-tree and the effectiveness of the tree representation in the database.

We verified that an R-tree in a relational database is useful for spatial metadata by comparing MAQR-tree performance with a non-tree implementation that relies heavily on the underlying relational database. For this pure relational implementation, we used the database schema shown in figure 5. Here, MBR coordinates are stored as separate integer fields in the database, making them directly available in SQL queries. Figure 6 shows an SQL implementation of a spatial intersection query using this schema. Indexes were built for all fields.

To carefully examine the advantage of the MAQR-tree over the pure relational implementation, we performed the comparison on four different datasets. We generate these datasets randomly as described before, but with different values for $P$, which determines the maximum size of replicas. This allows us to control the average number of replicas that intersect the query region in the different datasets. The advantage of the MAQR-tree query over pure relational query is presented in table 5 .

In table 5, we describes the average number of replicas returned per representative query on each dataset. And the average speedup is calculated by averaging the ratios of MAQR-tree query performance to that of pure relational for each query. We observed that the MAQR-tree query can be up to 8.13 times quicker than the pure relational implementation.

The reason for the advantages is very likely the multi-dimensional nature of the MAQR-tree. For pure relational implementation, even if an index can be used to quickly find the set of replicas with appropriate $x$ values, a $y$ index can not help with pruning this set further because it contains all the replicas in the database. A time consuming exhaustive search is therefore necessary. In contrast, the MAQR-tree query is able to drastically prune the 
set of replicas under consideration as it encounters multidimensional MBRs at each level of the tree, improving performance enormously.

Table 5 also shows that the MAQR-tree has the greatest advantage when the number of replicas returned is smaller. These more selective queries exhibit more aggressive pruning while traversing the tree, which enhances performance.

\begin{tabular}{|c|c|c|c|c|c|c|}
\hline $\begin{array}{c}\text { Node_id } \\
\text { (int) }\end{array}$ & $\begin{array}{c}\text { num_child } \\
\text { (int) }\end{array}$ & $\begin{array}{c}\text { (int) } \min \\
\text { (int }\end{array}$ & $\begin{array}{c}\text { y_min } \\
\text { (int) }\end{array}$ & $\begin{array}{c}\text { x_max } \\
\text { (int) }\end{array}$ & $\begin{array}{c}\text { y_max } \\
\text { (int) }\end{array}$ & $\begin{array}{c}\text { address } \\
\text { (varchar) }\end{array}$ \\
\hline 0 & 3 & 0 & 0 & 4 & 6 & null \\
\hline$\ldots \ldots$ & $\ldots \ldots$ & $\ldots \ldots$ & $\ldots \ldots$ & $\ldots \ldots$ & $\ldots \ldots .$. & $\ldots . .$. \\
\hline 2 I & 0 & I & I & 2 & 6 & "gsiftp://nodel..../d8.bin" \\
\hline
\end{tabular}

Figure 7: Alternative representation of the improved GTR-tree for the tree in figure 3. Components of MBRs are stored as integers.

We also tested the insertion cost with the two implementations. The pure relational implementation relies entirely on the underlying database to support insertion. For the MAQR-tree, we must perform several steps in order to insert a replica. Once the proper tree location has been determined, all siblings (sharing the same parent) after this location must increment its node id by one in order to make space for the insertion. In the rare case that no space is available, a node split operation must be performed [46], taking roughly 100 milliseconds in our tests. Next, the parent and ancestor MBRs are updated to account for the new node.

We tested MAQR-tree insertions with various groups of replica MBRs, including cubic MBRs of $50^{3}, 100^{3}, 200^{3}$, and $300^{3}$. We inserted these cube replicas into different regions of the entire domain. It takes an average of 30 milliseconds to insert one replica into a MAQR-tree. The pure relational approach is slightly quicker, taking an average of 18 milliseconds to insert, which is 1.67 times faster than the MAQR-tree. Overall, the MAQR-tree approach is more suitable for both scientific database and replica selection applications, which involves intensive queries and relatively less updates.

We evaluated the choice of table schemas used to represent MAQR-trees. We compared the performance of the schema shown in figure 7, with the schema presented in figure 3(c). We observed that MAQR-tree query performance with the former schema outperforms the latter by only $7.1 \%$ on average when using the MySQL backend. However, the schema shown in figure 7 makes the server code inflexible when dealing with n-dimensional 


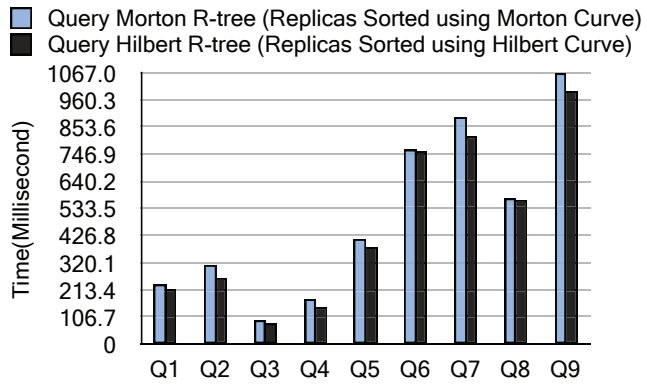

Figure 8: Morton R-tree compared with Hilbert R-tree. The Hilbert R-tree performance is $6.7 \%$ better than the MAQR-tree query performance on average.

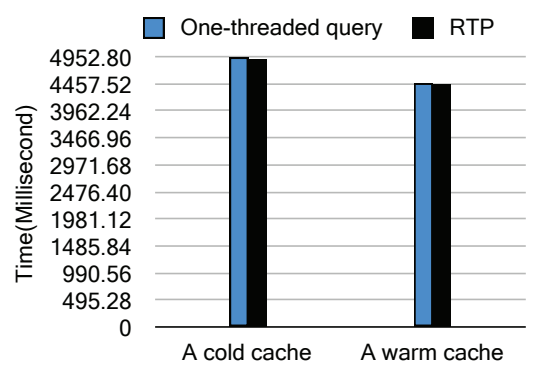

Figure 9: Average MAQR-tree query time without backend query aggregation, in which RTP is less effective, improving the query performance less than $1 \%$.

datasets. We have to change this table schema and the server code for different dimensional abilities. In contrast, the MAQR-tree representation shown in figure 3(c) requires no change. Furthermore, our RTP is more effective with the schema shown in figure 3(c) than that in figure 7, as discussed in section 7.3. Therefore, in our experiments we use the MAQR-tree representation shown in figure $3(\mathrm{c})$, unless otherwise noted.

\subsection{Morton R-tree}

We evaluated the effect of introducing the use of the Morton Space-filling curve to the MAQR-tree implementation, as described in section 6.3.1, and found that adding this technique improves performance by more than 30 times on average. Our preliminary experiments show that the Hilbert R-tree outperforms the Morton R-tree by a further $6.7 \%$ on dataset Data1 in table 5 , presented in figure 8. Although results presented in this paper use the Morton curve, we expect slightly better query performance in future by the applying Hilbert curve in the R-tree construction. In this test, we used a table-based algorithm to compute the Hilbert values for 3D MBRs, which is described in papers [29][47].

By associating each MBR with a Morton value, spatially adjacent replicas are mapped to similar Morton values. Therefore, they can be grouped into the same or neighboring parent tree nodes. In this way, the Morton value helps the enclosing MBRs to stay local, resulting in reduced "dead space" and possible overlaps in the tree node [46]. In short, the enclosing MBR is more efficiently used, and the constructed R-tree is more compact. This de- 


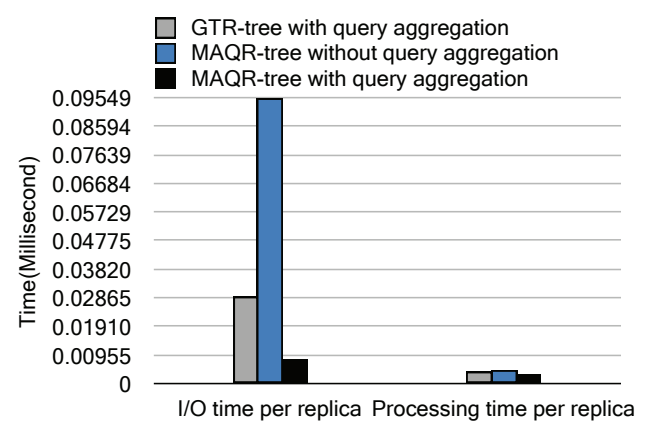

Figure 10: Average I/O time and processing time per replica measured with a warm cache. We find that backend query aggregation reduces I/O time per replica by a factor of 11.2 with a warm cache.

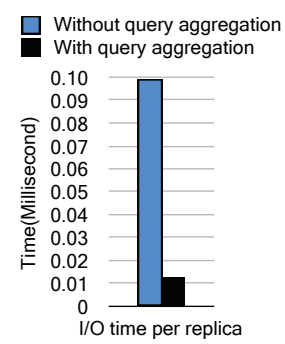

Figure 11: Average I/O time per replica in MAQR-tree measured with a cold cache. We find that backend query aggregation reduces I/O time per replica by $87.4 \%$ with a cold cache.

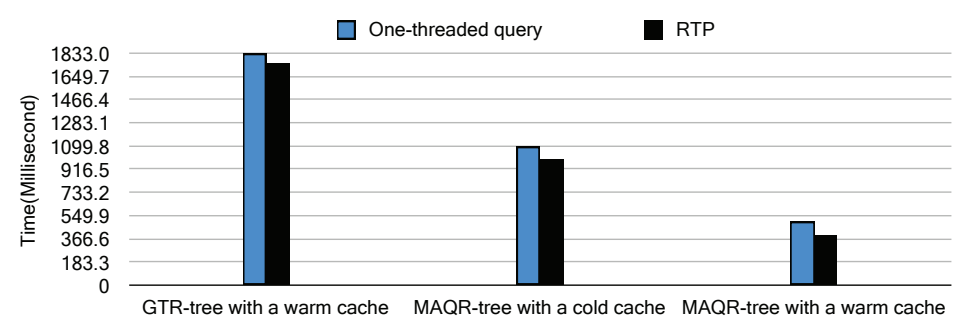

Figure 12: Average query time using backend query aggregation, in which RTP is more effective. RTP further improves MAQR-tree query performance by $9.4 \%$ under a cold cache, and by $23 \%$ under a warm cache. It also improves the GTR-tree performance by $4 \%$ under a warm cache.

creases the number of nodes that must be examined for intersection, thereby improving performance.

To further analyze the benefits of the Morton curve, we dumped each level of the MAQR-tree into a separate text file for later examination. We observed that the enclosing MBRs of internal nodes are more disjoint, with less overlap between neighboring tree nodes after the Morton Space-filling curve is introduced. For example, before using the morton curve it is observed that most MBRs on the second level of the tree fill up the entire dataset, which means they are much less effective in pruning the search during the R-tree query. In contrast, each of these level 2 MBRs covers only a local region of the entire dataset after the morton curve is used. 


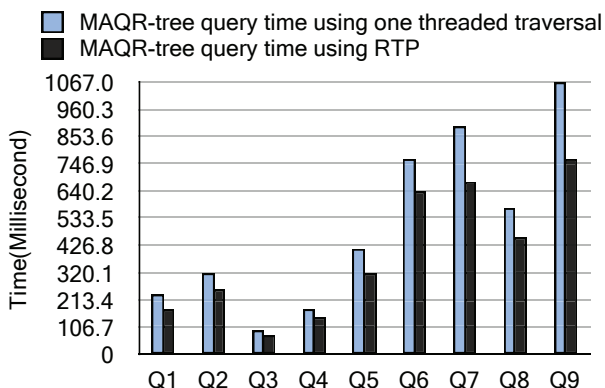

Figure 13: RTP compared with single threaded traversal using backend query aggregation under a warm cache for nine queries. RTP improves performance by $23 \%$ on average.

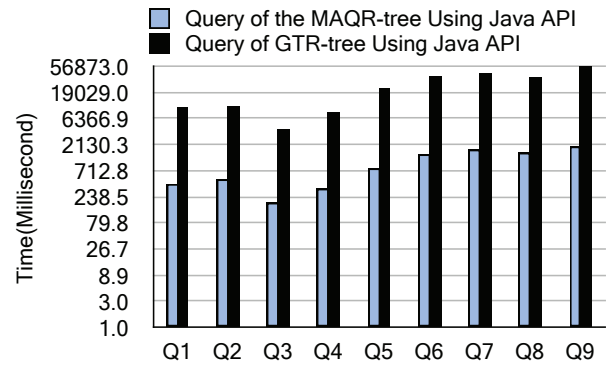

Figure 14: MAQR-tree query performance compared with that of GTR-tree. MAQR-tree outperforms GTR-tree 24.5 times on average. (Using Logarithmic Scale)

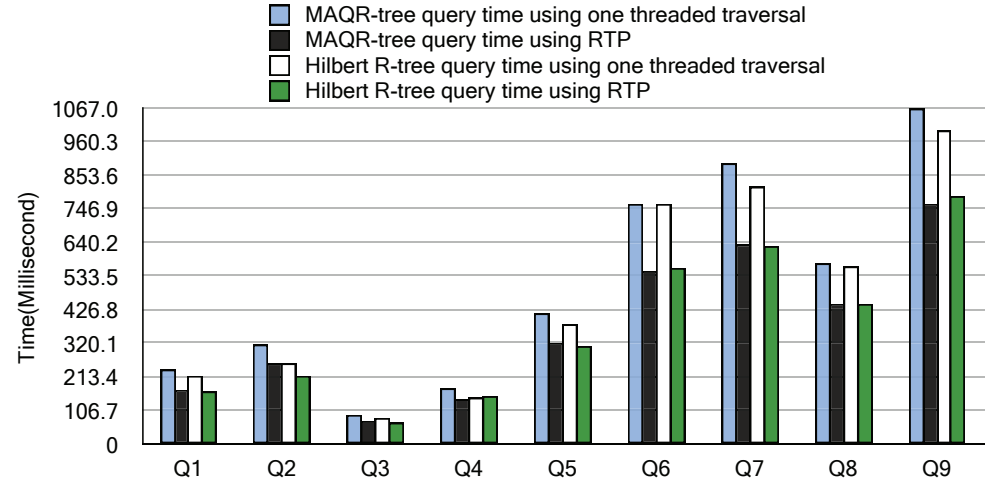

Figure 15: Effects of RTP on the query performance of the Hilbert R-tree and MAQRtree. Without RTP, the Hilbert R-tree is $6.7 \%$ quicker thanMAQR-tree on average, while with RTP enabled, the Hilbert R-tree and MAQR-Tree have nearly identical average performance. 


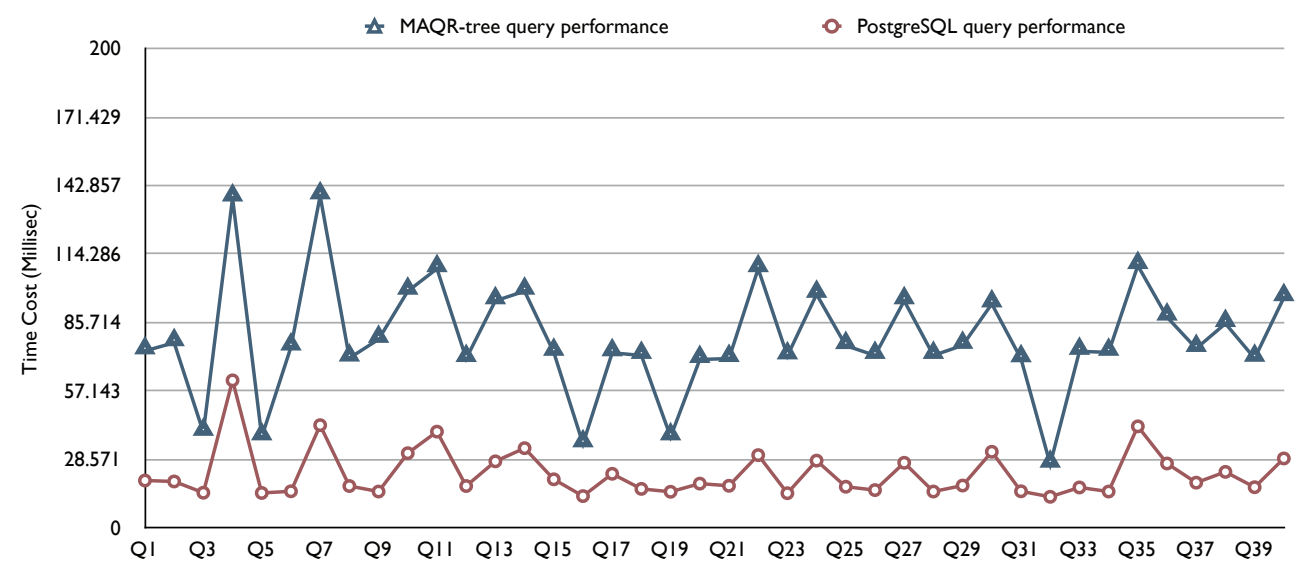

Figure 16: MAQR-tree query performance compared with that of PostgreSQL for a 3D dataset. The horizontal axis shows 40 random queries in the data domain. PostgreSQL using extension type Cube and GiST indexing outperforms our proposed MAQR-tree with RTP, probably due to function call overhead, as described in section 7.5.

\subsection{R-tree Prefetching (RTP)}

In order to analyze the behavior of RTP, we tested the average I/O time and processing time per replica under different circumstances. Given a tree node, the processing time per replica includes the time cost of the intersection test on the node's MBR, and enqueueing its children. The I/O time per replica is defined as the time cost to read into memory the information associated with each MAQR-tree node, such as the MBR and replica's physical address. This I/O time consists of two components. The hardware costs include disk latency, bus transfer costs, and the like. Software costs include costs associated with function calls or kernel routines.

It is noteworthy that in this section we only compared the representations of the MAQR-tree and the GTR-tree, in terms of I/O and processing time per replica. We tested the efficiency of both the GTR-tree and MAQRtree representations using the modified Globus RLS code from the MAQRtree. In section 7.4, we compare the MAQR-tree with the entire GTR-tree implementation that is built upon unmodified Globus.

\subsubsection{Effect of Backend Query Aggregation}

Our initial implementation of RTP did not use backend query aggregation. Unfortunately, we found that RTP alone was less effective with either a 
cold or warm cache, as shown in figure 9. Here, the overhead associated with the multithreaded implementation overwhelms any potential benefit. We addressed this shortcoming with backend query aggregation, which amortizes query costs over a collection of what would otherwise be separate queries. Comparing MAQR-tree single threaded query performance with and without this feature activated demonstrates an average $8 \mathrm{x}$ performance improvement for Backend Query Aggregation. Figure 12 shows that this strategy was effective, and allows RTP to demonstrate significant performance improvements. We use backend query aggregation with RTP in all following experiments, except where otherwise noted.

Backend Query aggregation is far more effective in reducing I/O time per replica with a warm cache than with a cold cache, which is perhaps counterintuitive. However, our results show that it reduces I/O time per replica by $87.4 \%$ with a cold cache and by a stunning factor of 11.2 with a warm cache, as shown in figure 11 and figure 10. With a cold cache, disk access is the dominant component of the I/O cost, while the software cost is minor. When collecting cold cache results we noticed that the first query always took far longer than the next several queries, due to the necessity of accessing the physical disk. This pattern repeats itself over time, but becomes progressively fainter due to the warming of the cache and prefetching by the filesystem. Query aggregation does not have significant effect on the hardware behavior, perhaps because a single disk access retrieves the data required for 50 queries even without aggregation. In contrast, with the warm cache case, software costs are the dominant component of I/O cost, and it is here that backend query aggregation shows substantial advantage because it pays these costs far less frequently.

\subsubsection{RTP Analysis}

RTP demonstrates the largest performance gain percentage when used with MAQR-tree under a warm cache, as shown in figure 12. The advantage of RTP over a single-threaded method in this environment is presented in figure 13. The query performance is improved on average by $23 \%$ for nine queries, after using RTP. The largest performance gain is $28 \%$ for query Q9 in which more than 26,400 intersecting replicas are returned.

We have already observed that RTP is less effective without backend query aggregation, as shown in figure 9. In our tests we used the same synchronization model regardless of whether backend query aggregation was used. However, backend query aggregation significantly coarsens the gran- 
ularity of synchronized queue access, allowing all children of a node that intersect the query MBR to be enqueued at once. Dequeueing behaves similarly. The net effect is to reduce the number of times queue operations are performed by a factor of out degree, which we have set to 50 in our experiments. Because queue access is guarded by a mutex, this reduces the impact of synchronization costs, an important component of the processing time.

When using two separate threads for I/O and computation, the best performance is obtained when I/O and computation costs per element are exactly equal, yielding twice the performance of the single threaded implementation. For this reason, RTP shows more performance improvement when the I/O time and the processing time per replica are well matched. Figure 10 shows that computation and I/O costs are best matched in the MAQR tree implementation using backend query aggregation on a warm cache, and we found RTP performance was improved by $23 \%$ over the single threaded implementation. In contrast, for the GTR-tree representation the I/O time per replica is 7.2 times larger than the processing time per replica, resulting in an average of only $4 \%$ performance improvement after using RTP. This also accounts for why RTP with MAQR-tree representation in figure 3(c) outperforms RTP with representation in figure 7 by $17 \%$.

In summary, RTP and backend query aggregation improve performance significantly, either with a cold cache or with a warm cache. Neither of these two testing scenarios are a perfect model of the real world, but two points should be made. First, cold caches are rare in practice because the filesystem cache will retain data until it has a reason to discard it, meaning that replica data could easily persist in filesystem memory for days. Second, scientific users often access the same "hot-spot" dataset regions repeatedly. In real world cases where the same (or nearby) data is repeatedly accessed, our warm cache results should prove an accurate indicator of performance.

\subsubsection{Effects of Space-filling Curves Under RTP}

We investigated the influence of RTP on the choice of space-filling curves. As described in section 7.2, without RTP enabled, the Hilbert R-tree outperforms the MAQR-tree (which uses a Morton curve) in query performance by $6.7 \%$. The Hilbert curve produces more compact nodes, which produces a performance increase during tree traversal because fewer nodes are visited. However, figure 15 shows that with RTP enabled, the advantage of the Hilbert curve disappears.

We explain this surprising fact by considering the filesystem cache as an 
important third factor. This cache views files as one dimensional, so when the application access pattern proceeds through the 1D file without jumping around, the filesystem cache's speculative prefetching is effective. Because the Hilbert curve jumps around in the 1D file space less frequently than the Morton curve, it usually has better performance. When RTP is added, however, our own SRLS is explicitly prefetching groups of required nodes, even when those groups span a gap in the 1D file. This makes the filesystem cache's speculative prefetching less useful, which in turn removes the Hilbert curve's advantage.

\subsection{Comparison with GTR-tree Implementation}

To make a fair comparison of the MAQR-tree with the GTR-tree, we tested on a single grid node using a same dataset. We compared the efficiency of the tree representation and associated techniques by sorting all replicas for both MAQR and GTR-tree with the same out degree. We observed the performance of the MAQR-tree is on average 24.5 times better than the GTR-tree, as shown in figure 14. We used the Java API for these tests, and excluded the time cost for establishing a secure connection. Results are shown on a logarithmic scale.

We attribute these speedups to the advantages of the MAQR-tree over the GTR-tree described in section 6.3.3 and section 5.3. We observed that the $\mathrm{I} / \mathrm{O}$ time cost per replica for MAQR-tree representation is more than 20 times quicker than that of GTR-tree mainly because the GTR-tree implementation did not apply backend query aggregation. In MAQR-tree, we also eliminated the local socket network communication between the server and the spatial query routine. These optimizations easily accounts for the improvements shown in the experimental results.

\subsection{Comparison with PostgreSQL Extension}

We compared our SRLS with an extension type Cube in PostgreSQL 9.4, one of the leading extensions to relational databases targeted toward highdimensional data. We randomly generated one million 3D replicas and compared query performance, shown in figure 16. The SRLS uses the MAQR-tree with RTP, while PostgreSQL uses a built-in GiST index [48]. PostgreSQL outperforms the MAQR-tree due to a larger number of SQL transaction overhead penalties.

During the test, we first submit a query to the SRLS client, which then sends the query to the SRLS server. In order to answer the spatial query 


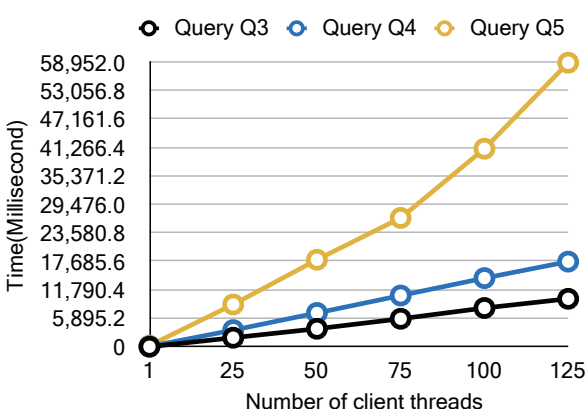

(a) Multi-client Experiment of the SRLS using RTP.

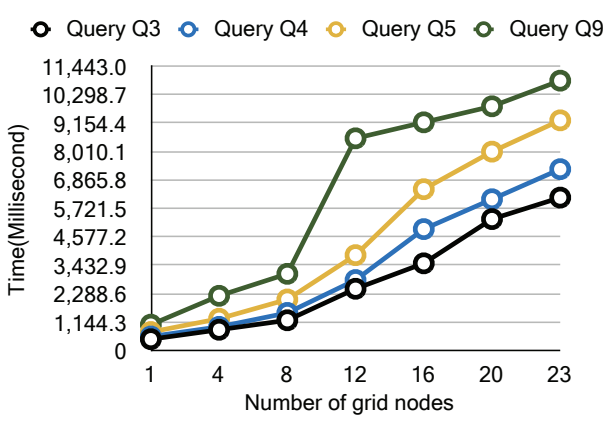

(b) Distributed query using one to twenty three nodes using SRLS with RTP enabled.

Figure 17: The multi-client test in (a) shows that server performance scales well as the number of simultaneous client threads increases. In (b) we see that performance scales well as we increase the number of server nodes and total number of replicas. In each test, the total number of replicas is the number of nodes multiplied by one million.

(an R-tree traversal), the SRLS server has to send multiple SQL queries to the backend relational database as tree traversal progresses. On the other hand, the PostgreSQL spatial extension answers the spatial query directly in one transaction. This explains why the two performance curves in figure 16 resemble each other, while the extra costs in the SRLS server shift its curve vertically.

However, the primary goal of this work is to add fast indexing for high dimensional data in existing middleware, the Replica Location Service (RLS). RLS primarily consists of three components - client tools, dedicated remote service APIs and backend data storage - a design widely accepted in the scientific data management community[14]. Because of the need to preserve this three-tier architecture, we implement the R-tree on top of the relational database already used by RLS. In contrast, Postgres is implementing its spatial data structure inside of the database, which improves performance. Investigating the use of PostgreSQL and its spatial extensions as part of SRLS is a topic for future work.

\subsection{Multi-client and Distributed Experiments}

We wrote a multi-threaded client program using our C API and the pthreads library. We conducted the multi-client tests with the server and clients on a same machine, on which our threaded client program allows us 
to specify how many client threads are created simultaneously. In the distributed query, initially we submitted queries on a client grid node at the University of Mississippi to a server node at the University of Chicago. We then increased the number of server nodes by adding a combination of at least two remote nodes and one local node.

The results of the multi-client and distributed query are shown in figure 17. Figure 17(a) shows that server performance scales well as the number of simultaneous client threads increases. In (b) we see that performance also scales well as we increase the number of server nodes and total number of replicas. Each server maintains one million replicas. With twenty three nodes we are handling twenty three million replicas, but execution time is less than ten times as long as for one million replicas. We observed a sharp increase in the query time for Q9 using between eight and twelve nodes in (b). This is due to adding the server nodes at the University of Hawaii and the larger amount of replicas returned, which are transferred via a network with latency more than twice higher. The average number of replicas intersected with each query on each grid node is shown in table 3.

\section{Conclusion}

Our eventual goal is a system where both data and computation are truly distributed. We envision applications where only a subvolume of a larger dataset is required for a computation, and where that subvolume may be available from various combinations of different sources. If we hope to provide more than simple batch computation for such applications, we must be able to rapidly identify the set of partial replicas that intersect with the required subvolume, and then choose an optimal combination of replicas to read from. This paper presents work that rapidly identifies the set of intersecting replicas in a distributed environment. We present two different designs for SRLS in a grid and many optimization techniques.

We identify two major contributions of this paper. First, to the best of our knowledge, this is the first proposal to implement a tree structure inside a relational database for metadata management. Using this design, complexity and development cycles of such a location service could be dramatically reduced by using the atomicity features in DBMS, while still providing high performance comparable to PostgreSQL. Second, we proposed R-tree prefetching. Through experiments, we provide deeper understanding with regard to why the R-tree prefetching is influenced by backend query 
aggregation? and why it affects the effectiveness of Space-filling curves? We believe the R-tree prefetching could be easily adapted to other systems that rely on tree traversal operations.

There are several avenues for future research. We may also continue our optimization efforts by replacing the database with our own storage module, giving greater control over disk behavior. The problem of selecting an optimal combination of partial replicas must also be addressed, along with the development of suitable performance metrics. Lastly, we would investigate the use of PostGIS or PostgreSQL and its spatial extensions as part of SRLS.

\section{Acknowledgment}

This work was supported by the National Science Foundation under grants CCF-0541239 and CRI-0855136. 
[1] S. Vazhkudai, S. Tuecke, I. Foster, Replica selection in the globus data grid, in: Proc. CCGRID '01, Published by the IEEE Computer Society, 2001, p. 106.

[2] A. Chervenak, E. Deelman, I. Foster, L. Guy, W. Hoschek, A. Iamnitchi, C. Kesselman, P. Kunszt, M. Ripeanu, B. Schwartzkopf, H. Stockinger, K. Stockinger, B. Tierney, Giggle: a framework for constructing scalable replica location services, in: Proceedings of the 2002 ACM/IEEE conference on Supercomputing, IEEE Computer Society Press, Baltimore, Maryland, 2002, pp. 1-17.

[3] A. Chervenak, N. Palavalli, S. Bharathi, C. Kesselman, R. Schwartzkopf, Performance and scalability of a replica location service, Proceedings of the 13th IEEE International Symposium on High Performance Distributed Computing (2004) 182-191.

[4] M. Cai, A. Chervenak, M. Frank, A peer-to-peer replica location service based on a distributed hash table, in: Proceedings of the 2004 ACM/IEEE conference on Supercomputing, IEEE Computer Society, 2004, p. 56.

[5] Globus.org, Globus Toolkit 5.0.3 RLS User's Guide.

URL http://www.globus.org/toolkit/docs/5.0/5.0.3/data/rls/ user/\#rlsUser

[6] W. Peng-fei, F. Yu, C. Bin, W. Xi, GloSDC: A Framework for a Global Spatial Data Catalog, in: Geoscience and Remote Sensing Symposium, 2008. IGARSS 2008. IEEE International, Vol. 2, IEEE, 2009.

[7] Y. Wei, L. Di, B. Zhao, G. Liao, A. Chen, Y. Bai, Y. Liu, The design and implementation of a grid-enabled catalogue service, in: International Geoscience and Remote Sensing Symposium, Vol. 6, 2005, p. 4224.

[8] L. Di, A. Chen, W. Yang, Y. Liu, Y. Wei, P. Mehrotra, C. Hu, D. Williams, The development of a geospatial data grid by integrating OGC web services with globus-based grid technology, Concurrency and Computation: Practice and Experience 20 (14) (2008) 1617-1635.

[9] A. Innman, G. Easson, V. Asper, et al., The effectiveness of using modis products to map sea surface oil, in: OCEANS 2010, IEEE, 2010, pp. 1-5. 
[10] P. J. Rhodes, X. Tang, R. D. Bergeron, T. M. Sparr, Iteration Aware Prefetching for Large Multidimensional Scientific Datasets, in: SSDBM'2005: Proc. of the 17th international conference on Scientific and statistical database management, Lawrence Berkeley Laboratory, Berkeley, CA, US, 2005, pp. 45-54.

[11] P. J. Rhodes, S. Ramakrishnan, Iteration Aware Prefetching for Remote Data Access, in: H. Stockinger, R. Buyya, R. Perrott (Eds.), Proc. 1st International Conference on e-Science and Grid Computing, 2005, pp. 279-286.

[12] O. O. Akande, P. J. Rhodes, Towards an efficient storage and retrieval mechanism for large unstructured grids, Future Generation Computer Systems (0) (2014) -. doi:http://dx.doi.org/10.1016/j.future.2014.10.024.

URL http://www.sciencedirect.com/science/article/pii/ S0167739X14002180

[13] Y. Gu, R. Grossman, UDT: UDP-based data transfer for highspeed wide area networks, Comput. Netw. 51 (7) (2007) 1777-1799. doi:http://dx.doi.org/10.1016/j.comnet.2006.11.009.

[14] J. Gray, D. T. Liu, M. Nieto-Santisteban, A. Szalay, D. J. DeWitt, G. Heber, Scientific data management in the coming decade, SIGMOD Rec. 34 (2005) 34-41. doi:http://doi.acm.org/10.1145/1107499.1107503. URL http://doi.acm.org/10.1145/1107499.1107503

[15] A. Chervenak, R. Schuler, M. Ripeanu, A. Amer, S. Bharathi, I. Foster, A. Iamnitchi, C. Kesselman, The globus replica location service: design and experience, Parallel and Distributed Systems, IEEE Transactions on 20 (9) (2009) 1260-1272.

[16] R. Rahman, R. Alhajj, K. Barker, Replica selection strategies in data grid, Journal of Parallel and Distributed Computing 68 (12) (2008) 1561-1574.

[17] K. Li, H. Wang, K. Cheng, T. Wu, Strategies Toward Optimal Access to File Replicas in Data Grid Environments, Journal of Information Science and Engineering 25 (3) (2009) 747-762. 
[18] Y. Zhao, Y. Hu, GRESS-a grid replica selection service, in: Proceedings of the 15th IASTED International Conference on Parallel and Distributed Computing and Systems (PDCS-2003), 2003.

[19] PostGIS Webpage [online, cited July 2011].

[20] S. Narayanan, T. Kurc, U. Catalyurek, J. Saltz, Database support for data-driven scientific applications in the grid, PPL-Parallel Processing Letters 13 (2) (2003) 245-272.

[21] S. Narayanan, U. Catalyurek, T. Kurc, V. Kumar, J. Saltz, A runtime framework for partial replication and its application for on-demand data exploration, in: High Performance Computing Symposium (HPC 2005), SCS Spring Simulation Multiconference, 2005.

[22] L. Weng, U. Catalyurek, T. Kurc, G. Agrawal, J. Saltz, Servicing range queries on multidimensional datasets with partial replicas, in: Cluster Computing and the Grid, 2005. CCGrid 2005. IEEE International Symposium on, Vol. 2, IEEE, 2005, pp. 726-733.

[23] A. Guttman, R-trees: A Dynamic Index Structure for Spatial Searching, in: SIGMOD '84: Proceedings of the 1984 ACM SIGMOD international conference on Management of data, ACM, New York, NY, USA, 1984, pp. 47-57. doi:http://doi.acm.org/10.1145/602259.602266.

[24] F. Ramsak, V. Markl, R. Fenk, M. Zirkel, K. Elhardt, R. Bayer, Integrating the ub-tree into a database system kernel, in: A. E. Abbadi, M. L. Brodie, S. Chakravarthy, U. Dayal, N. Kamel, G. Schlageter, K.-Y. Whang (Eds.), VLDB 2000, Proceedings of 26th International Conference on Very Large Data Bases, September 10-14, 2000, Cairo, Egypt, Morgan Kaufmann, 2000, pp. 263-272.

[25] I. Kamel, C. Faloutsos, Hilbert R-tree: An improved R-tree using fractals, in: Proceedings of the International conference on Very Large Databases, 1994, pp. 500-509.

[26] J. L. Bentley, Multidimensional binary search trees used for associative searching, Commun. ACM 18 (1975) 509-517. 
[27] S. Berchtold, D. Keim, H. Kriegel, The X-tree: An index structure for high-dimensional data, Readings in multimedia computing and networking 12 (2002) 451.

[28] V. Gaede, O. Günther, Multidimensional access methods, ACM Computing Surveys (CSUR) 30 (2) (1998) 170-231.

[29] C. Faloutsos, S. Roseman, Fractals for secondary key retrieval, in: Proceedings of the eighth ACM SIGACT-SIGMOD-SIGART symposium on Principles of database systems, ACM, 1989, pp. 247-252.

[30] Y. Fang, M. Friedman, G. Nair, M. Rys, A. Schmid, Spatial indexing in microsoft SQL server 2008, in: Proceedings of the 2008 ACM SIGMOD international conference on Management of data, ACM, 2008, pp. 12071216.

[31] P. Ganesan, B. Yang, H. Garcia-Molina, One torus to rule them all: multi-dimensional queries in p2p systems, in: Proceedings of the 7th International Workshop on the Web and Databases: colocated with ACM SIGMOD/PODS 2004, WebDB '04, ACM, New York, NY, USA, 2004, pp. 19-24.

[32] C. Luk, T. Mowry, Compiler-based prefetching for recursive data structures, in: ACM SIGOPS Operating Systems Review, Vol. 30, ACM, 1996, pp. 222-233.

[33] C. Luk, T. Mowry, Automatic compiler-inserted prefetching for pointerbased applications, IEEE Transactions on Computers 48 (2) (1999) 134141.

[34] J. Rao, K. Ross, Making b+-trees cache conscious in main memory, in: ACM SIGMOD Record, Vol. 29, ACM, 2000, pp. 475-486.

[35] R. Patterson, G. Gibson, E. Ginting, D. Stodolsky, J. Zelenka, Informed prefetching and caching, in: Proceedings of the fifteenth ACM symposium on Operating systems principles, ACM, 1995, pp. 79-95.

[36] S. Chen, P. Gibbons, T. Mowry, Improving index performance through prefetching, Vol. 30, ACM, 2001. 
[37] S. Chen, P. Gibbons, T. Mowry, G. Valentin, Fractal prefetching b+trees: Optimizing both cache and disk performance, in: Proceedings of the 2002 ACM SIGMOD international conference on Management of data, ACM, 2002, pp. 157-168.

[38] H. Kang, J. Kim, D. Kim, K. Han, An Extended R-Tree Indexing Method Using Selective Prefetching in Main Memory, Computational Science-ICCS 2007 (2007) 692-699.

[39] C. Kim, J. Chhugani, N. Satish, E. Sedlar, A. Nguyen, T. Kaldewey, V. Lee, S. Brandt, P. Dubey, Fast: fast architecture sensitive tree search on modern cpus and gpus, in: Proceedings of the 2010 international conference on Management of data, ACM, 2010, pp. 339-350.

[40] P. Lindstrom, Out-of-core construction and visualization of multiresolution surfaces, in: Proceedings of the 2003 symposium on Interactive 3D graphics, I3D '03, ACM, New York, NY, USA, 2003, pp. 93-102.

[41] P. Sulatycke, K. Ghose, A fast multithreaded out-of-core visualization technique, in: Parallel and Distributed Processing, 1999. 13th International and 10th Symposium on Parallel and Distributed Processing, 1999. 1999 IPPS/SPDP. Proceedings, IEEE, 2002, pp. 569-575.

[42] Y. Tian, P. J. Rhodes, The Globus Toolkit R-tree for Partial Spatial Replica Selection, in: Proceedings of the 2010 11th IEEE/ACM International Conference on Grid Computing, IEEE, Brussels, Belgium, 2010, pp. 169-176.

[43] Y. Tian, P. J. Rhodes, A fast location service for partial spatial replicas, in: Proceedings of the 2011 IEEE/ACM 12th International Conference on Grid Computing, IEEE Computer Society, 2011, pp. 190-197.

[44] Globus.org, Replica Location Service RPC Protocol Description (2003). URL http://www.globus.org/toolkit/docs/5.0/5.0.3/data/rls/ developer/rpcprotocol.pdf

[45] S. Leutenegger, M. Lopez, J. Edgington, STR: A simple and efficient algorithm for R-tree packing, in: Data Engineering, 1997. Proceedings. 13th International Conference on, 1997, pp. 497-506. 
[46] N. Beckmann, H. Kriegel, R. Schneider, B. Seeger, The R*-tree: an efficient and robust access method for points and rectangles, ACM SIGMOD Record 19 (2) (1990) 322-331.

[47] J. Lawder, P. King, Using state diagrams for hilbert curve mappings, International journal of computer mathematics 78 (3) (2001) 327-342.

[48] J. Hellerstein, J. Naughton, A. Pfeffer, Generalized search trees for database systems, Readings in database systems (1998) 101. 